open Magnetically tunable Mie

SUBJECT AREAS:

MATERIALS FOR OPTICS

OPTICAL MATERIALS AND

STRUCTURES

OPTICAL PHYSICS

Received

14 September 2014

Accepted

23 October 2014

Published

11 November 2014

Correspondence and requests for materials should be addressed to

J.H.X. (jhxiao@bupt.

edu.cn);M.L. (mlei@

bupt.edu.cn) or J.Z.

(zhouji@mail.tsinghua.

edu.cn) \title{
resonance-based dielectric metamaterials
}

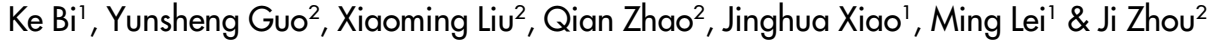

\begin{abstract}
'State Key Laboratory of Information Photonics and Optical Communications \& School of Science, Beijing University of Posts and Telecommunications, Beijing 100876, China, ${ }^{2}$ State Key Laboratory of New Ceramics and Fine Processing, School of Materials Science and Engineering, Tsinghua University, Beijing 100084, China.
\end{abstract}

Electromagnetic materials with tunable permeability and permittivity are highly desirable for wireless communication and radar technology. However, the tunability of electromagnetic parameters is an immense challenge for conventional materials and metamaterials. Here, we demonstrate a magnetically tunable Mie resonance-based dielectric metamaterials. The magnetically tunable property is derived from the coupling of the Mie resonance of dielectric cube and ferromagnetic precession of ferrite cuboid. Both the simulated and experimental results indicate that the effective permeability and permittivity of the metamaterial can be tuned by modifying the applied magnetic field. This mechanism offers a promising means of constructing microwave devices with large tunable ranges and considerable potential for tailoring via a metamaterial route.

(1) lectromagnetic metamaterials with exotic physic properties have stimulated tremendous fundamental and practical interests during past decade ${ }^{1-5}$. Metamaterials are a class of materials in which subwavelength features, rather than the constituent materials, control the macroscopic electromagnetic properties ${ }^{6}$. Up to now, most of present metamaterials are constructed by using artificial metallic microstructures such as split ring resonator (SRR) or wire, whose size and periodicity are far smaller than the operating wavelengths so that the response of metamaterial to electromagnetic wave can be interpreted by using effective parameters ${ }^{7-10}$. The unusual electromagnetic properties of metamaterials originate from artificial structure rather than being inherited directly from the materials, which opens a way to design material with more freedom ${ }^{11-13}$.

Recently, Mie resonance-based metamaterials have been theoretical and experimentally studied ${ }^{14-18}$. The electromagnetic wave interaction of dielectric particles with relative high permittivity can exhibit a strong magnetic or electric resonance. From the oscillation of resulting magnetic or electric dipole, negative permeability or permittivity can be produced ${ }^{19}$. Compared to SRR and related structures, Mie resonance-based metamaterial can be expected to realize low loss and isotropic properties because it does not suffer from conductive currents and capacitance gap ${ }^{20}$. It is well known that the permeability and permittivity of the materials are key factors for the propagation of the electromagnetic wave. In order to decrease the number of parts, materials for microwave devices are required to have field dependence properties and one of the easiest ways to satisfy this need is to find materials where the parameters show magnetic DC bias dependence. However, the effective parameters cannot be tuned as a result of the settled characteristic frequency band in artificial metallic structure or Mie resonance-based metamaterials, since the size and structure of those metamaterials are confirmed.

Here, we report a magnetically tunable metamaterials based on the coupling of the Mie resonance of dielectric cube and ferromagnetic precession of ferrite cuboid. The electromagnetic properties of the metamaterials were investigated by simulations and experiments. Our results demonstrate the effective permittivity and permeability of the metamaterial can be tuned by applied magnetic field.

\section{Results}

Design and characterization of the magnetically tunable Mie resonance-based dielectric metamaterial. Figure 1a shows the schematic diagram of the magnetically tunable Mie resonance-based dielectric metamaterial. The unit cell of the metamaterial is composed of one dielectric cube and two ferrite cuboids surrounded by air. Dielectric particles support an electric and magnetic dipole response attributable to Mie resonances. The effective permittivity and permeability of an array of dielectric spherical particles embedded 


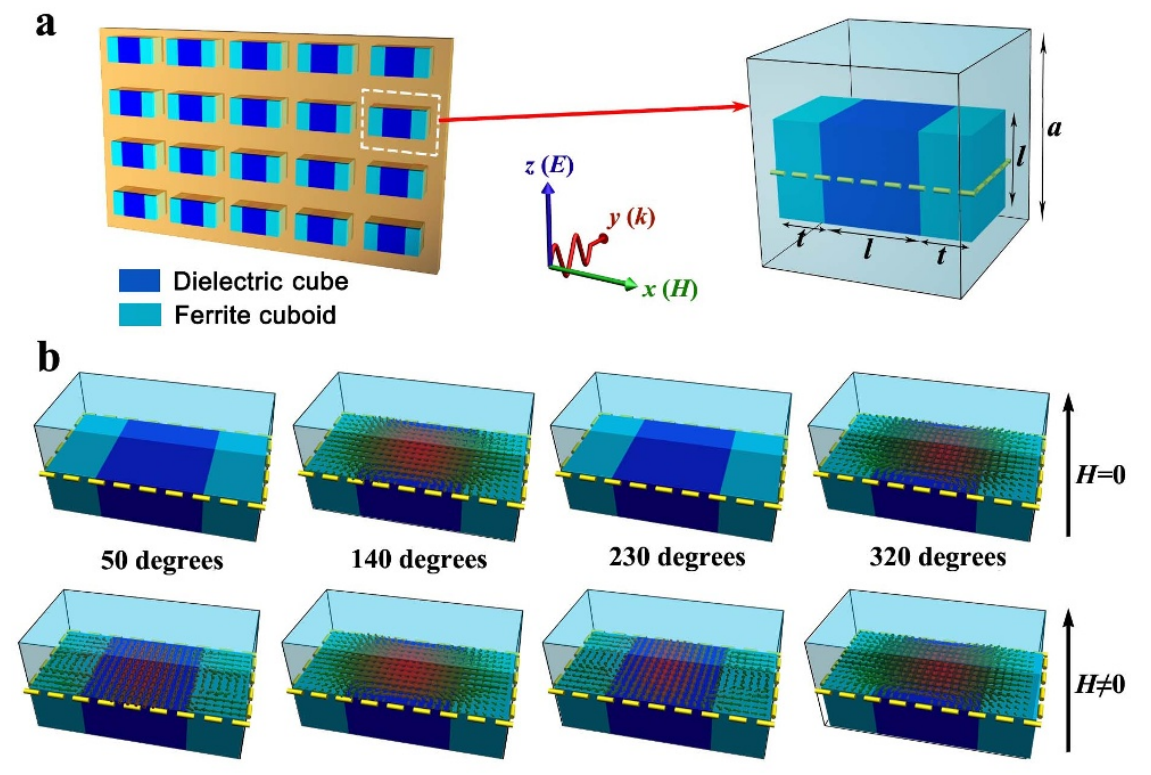

Figure 1 Schematic diagram of the magnetically tunable Mie resonance-based dielectric metamaterial. (a) The metamaterial and the unit cell consisting of one dielectric cube and two ferrite cuboids. The propagation of the incident electromagnetic wave is along the $y$ axis, and the electric field and magnetic field are along the $z$ and $x$ axes, respectively. The bias magnetic field is applied in the $z$ direction. (b) Magnetic field distributions at Mie resonance frequency illustrating the effect of applied magnetic field on Mie resonance. Four maps with different phase (50, 140, 230 and 320 degrees) are chosen to indicate the dynamic changes of magnetic field distributions in the unit cell. The upper and lower maps show the magnetic field distributions for the unit cell without and with applied magnetic field, respectively.

in a matrix can be expressed as follows ${ }^{21}$ :

$$
\begin{aligned}
& \varepsilon_{e f f}=\varepsilon_{1}\left(1+\frac{3 v}{\frac{F(\theta)+2 K_{e}}{F(\theta)-K_{e}}-v}\right), \\
& \mu_{e f f}=\mu_{1}\left(1+\frac{3 v}{\frac{F(\theta)+2 K_{m}}{F(\theta)-K_{m}}-v}\right),
\end{aligned}
$$

where

$$
F(\theta)=\frac{2(\sin \theta-\theta \cos \theta)}{\left(\theta^{2}-1\right) \sin \theta+\theta \cos \theta}, \theta=k_{0} r_{0} \sqrt{\varepsilon_{2} \mu_{2}}, k_{0}=2 \pi / \lambda, K_{e}=
$$
$\varepsilon_{1} / \varepsilon_{2}, K_{m}=\mu_{1} / \mu_{2}, v=\frac{4}{3} \pi\left(\frac{r_{0}}{p}\right)^{3}$ is the volume fraction of dielectric particles, $r_{0}$ and $p$ are the particle radius and the lattice constant, respectively; $\varepsilon_{1}$ and $\mu_{1}$ are the permittivity and permeability of the background matrix, respectively; $\varepsilon_{2}$ and $\mu_{2}$ are the permittivity and permeability of the dielectric particles, respectively. From Eqs. (1) and (2), we observe that the effective permittivity $\varepsilon_{\text {eff }}$ and permeability $\mu_{\text {eff }}$ can be not only influenced by the permittivity $\varepsilon_{2}$ and the permeability $\mu_{2}$ of the dielectric particles, but also affected by the permittivity $\varepsilon_{1}$ and the permeability $\mu_{1}$ of the background matrix. Meanwhile, by interacting with the magnetic field of an electromagnetic wave, ferromagnetic precession can arise in ferrite under an applied magnetic field. The effective permeability of the ferrite around the frequency area of ferromagnetic resonance can be expressed as follows ${ }^{22}$ :

$$
\mu_{3}=1-\frac{F \omega_{\mathrm{mp}}^{2}}{\omega^{2}-\omega_{\mathrm{mp}}^{2}-i \Gamma(\omega) \omega}
$$

where $\Gamma(\omega)=\left[\omega^{2} /\left(\omega_{\mathrm{r}}+\omega_{\mathrm{m}}\right)+\omega_{\mathrm{r}}+\omega_{\mathrm{m}}\right] \alpha ; \omega_{\mathrm{mp}}=\sqrt{\omega_{\mathrm{r}}\left(\omega_{\mathrm{r}}+\omega_{\mathrm{m}}\right)}$; $\omega_{\mathrm{m}}=4 \pi M_{\mathrm{s}} \gamma ; \omega_{\mathrm{r}}=\gamma H ; \alpha$ is the damping coefficient of ferromagnetic precession; $\gamma$ is the gyromagnetic ratio; $F=\omega_{\mathrm{m}} / \omega_{\mathrm{r}} ; \omega_{\mathrm{m}}$ and $\omega_{\mathrm{r}}$ are the characteristic frequency and ferromagnetic resonance frequency of the ferrite, respectively; $M_{\mathrm{s}}$ is the saturation magnetization caused by the applied magnetic field; and $H$ is the applied magnetic field. According to Eq. (3), the permeability of the ferrite can be tuned by adjusting the applied magnetic field. Because ferrite is one of the two important parts in this metamaterial, the effective permeability of the metamaterial can be influenced by the permeability of the ferrite cuboids, which means that the effective permeability of the metamaterial can be tuned by adjusting the applied magnetic field. To simplify fabrication, cubes rather than spheres were used as the dielectric particles to prepare the metamaterial.

In order to understand the influence of applied magnetic field on the electromagnetic properties of the metamaterial, the dynamic magnetic field distributions of the unit cell were simulated. Figure $1 \mathrm{~b}$ shows the magnetic field distributions for the unit cell with or without applied magnetic filed. Four maps with different phase (50, 140, 230 and 320 degrees) are chosen to indicate the dynamic changes of magnetic field distributions in the unit cell. For the unit cell without applied magnetic field, the dielectric cube plays a key role in interacting with electromagnetic wave, and the ferrite cuboids do not interacts with electromagnetic wave due to they have not been magnetized. At a certain frequency (Mie resonance frequency, $11.37 \mathrm{GHz}$ ), the magnetic field distributions in the $x y$-plane (the upper four maps in Fig. 1b) show a typical magnetic Mie-resonance mode. First, the magnetic fields are mainly localized in the cubes at the Mie-resonance frequency ${ }^{16}$. Second, it can be seen that the displacement currents at 50 and 230 degrees are nearly disappeared and that at 140 and 320 degrees localize in the center of the unit cell. The direction of the linearly polarized displacement currents at 140 degrees is opposite to that at 320 degrees along the $x$ axis, which exhibits a magnetic dipole characteristic. Ferrite can be magnetized in presence of a certain external magnetic field. When a bias magnetic field (1500 Oe) is applied along $z$ direction, the ferromagnetic precession takes place in the ferrite cuboids by interacting with the electromagnetic wave, which could influence the Mie resonance of the dielectric cube. The magnetic field distributions at $11.60 \mathrm{GHz}$ for 
the unit cell with applied magnetic filed are shown in the lower four maps of Fig. 1b. The magnetic field distributions at 140 and 320 degrees for the unit cell with applied magnetic filed are the same as that for the unit cell without applied magnetic filed. However, at 50 and 230 degrees, the magnetic field distributions for the unit cell with and without applied magnetic filed are much different. According to the dynamic simulation, when a bias magnetic field is applied along $z$ direction, the linearly polarized displacement currents in dielectric cube rotate clockwise and that in ferrite cuboids rotate anticlockwise. At 140 and 320 degrees, the behavior of the displacement currents exhibits a magnetic dipole characteristic. At 50 and 230 degrees, the magnetic field distributions in ferrite cuboids show a vortical pattern, which exhibits an electric dipole characteristic. On the basis of the analysis of the magnetic field distributions for the unit cell with or without applied magnetic filed, one can predict that the applied magnetic filed not only influences the permeability of the metamaterial, but also affects the permittivity of the metamaterial.

Numerical simulations. Figure 2a shows the simulated transmission spectra for the unit cell of the metamaterial under a series of applied magnetic fields $H$. A transmission dip appears at $11.37 \mathrm{GHz}$ in the absence of an applied magnetic field, which corresponds to the magnetic Mie-resonance ${ }^{20}$. When a magnetic field of $H=500 \mathrm{Oe}$ is applied, one can see that two transmission dips appear at $11.24 \mathrm{GHz}$ and $11.48 \mathrm{GHz}$, respectively. This indicates that the electromagnetic properties of the metamaterial can be affected by the applied magnetic field. When $H$ is increased from 500 Oe to 2000 Oe, two transmission dips move to higher frequency region, which exhibits a magnetically tunable behavior. When $H$ is increased to $2500 \mathrm{Oe}$, the third transmission dip appears at $8.29 \mathrm{GHz}$, corresponding to the ferromagnetic resonance of the ferrite cuboids $^{23}$. The effective permittivity and permeability of the unit cell of the metamaterial under the same series of applied magnetic fields $H$ was extracted from the simulated scattering parameters using a well-developed retrieval algorithm ${ }^{24-26}$. Figure $2 \mathrm{~b}$ shows the dependence of the calculated effective permeability on frequency. For the unit cell without applied magnetic field, one remarkable Lorentztype dispersion occurs in the range of 11-12 GHz. When a certain magnetic field is applied, instead of one dispersion, two or three frequency dispersions appear in the range of 11-12 GHz, which also exhibit magnetic resonance characteristic. The resonance frequency increases as $H$ increases, consistent with the behavior observed in Fig. 2a. In addition, between the magnetic resonance frequency and the magnetic plasma frequency, the value of the effective permittivity is negative. This characteristic makes this metamaterial suitable for applications in negative refractive index materials. Figure $2 \mathrm{c}$ shows the dependence of the calculated effective permittivity on frequency. Corresponding to each Lorentz-type dispersion for the effective permeability shown in Fig. 2b, a concurrent anti-resonance state of the effective permittivity appears due to the periodicity effect ${ }^{27}$. Besides that, the electric resonance frequency increases as $H$ increases. From the above analysis, both the effective permittivity and permeability of the metamaterial can be tuned by applied magnetic field.

Microwave measurement system. To confirm the results of the above simulations, experimental investigations of the electromagnetic properties of this metamaterial were conducted. The photograph of the microwave measurement system and the metamaterial are shown in Fig. 3. The upper and lower metal plates form the planar waveguide, which restrict the polarization of the electric field to lie uniformly along the $\mathrm{z}$ direction and further restrict propagation to the $x-y$ plane. Two horn antennas connected to the output and input ports of the vector network analyzer (Agilent ENA5071C) are installed at both ends of planar waveguide (between the upper and lower metallic plates) as the waveguide adapter to excite and receive a plane wave $(8-12 \mathrm{GHz})$. The sample is placed
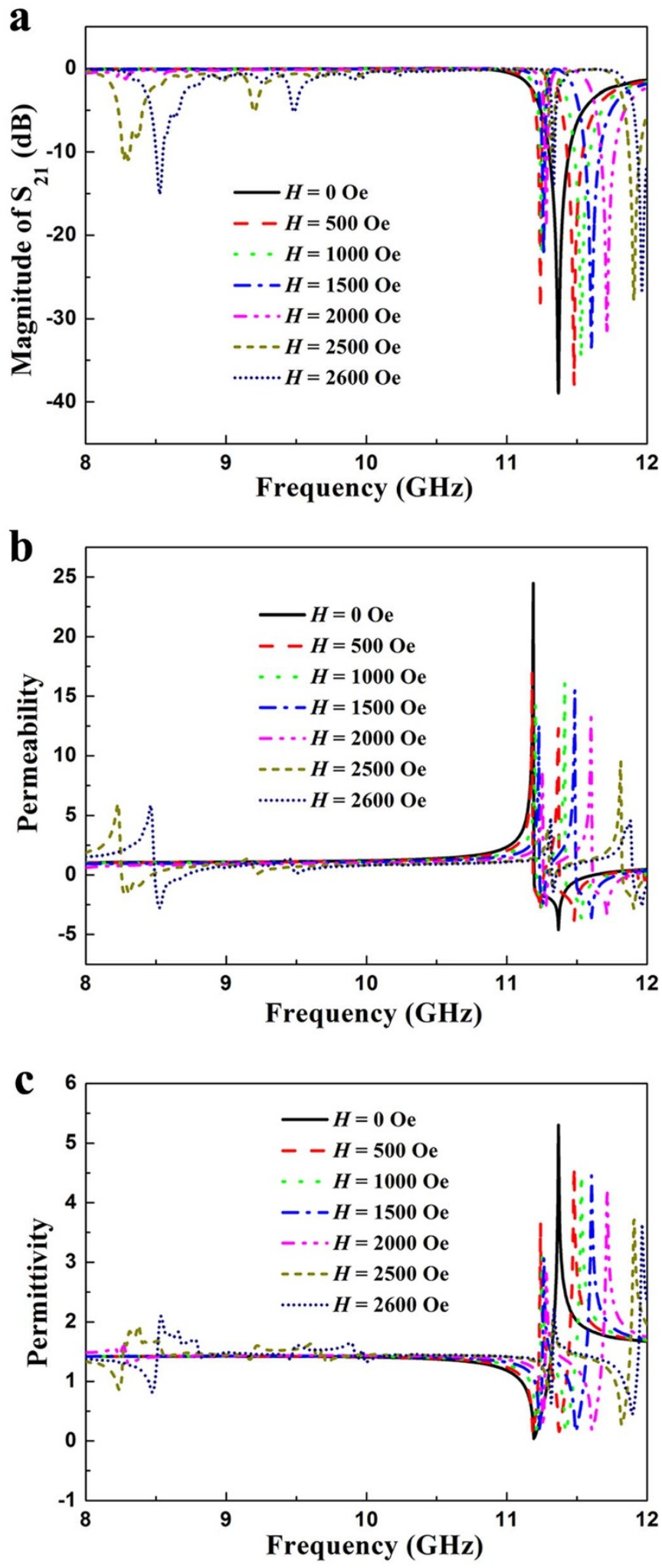

Figure $2 \mid$ Simulation demonstrating the magneto-tunable behavior. (a) Simulated transmission spectra for the unit cell of the metamaterial under a series of applied magnetic fields $H$. Real parts of the effective (b) permeabilities and (c) permittivities retrieved from the simulated scattering parameters under a series of applied magnetic fields $H$.

between two horn antennas. The propagation of the incident electromagnetic wave is along the $y$ axis, and the electric field and magnetic field are along the $z$ and $x$ axes, respectively. The bias magnetic field is generated by an electromagnet in the $z$ direction 


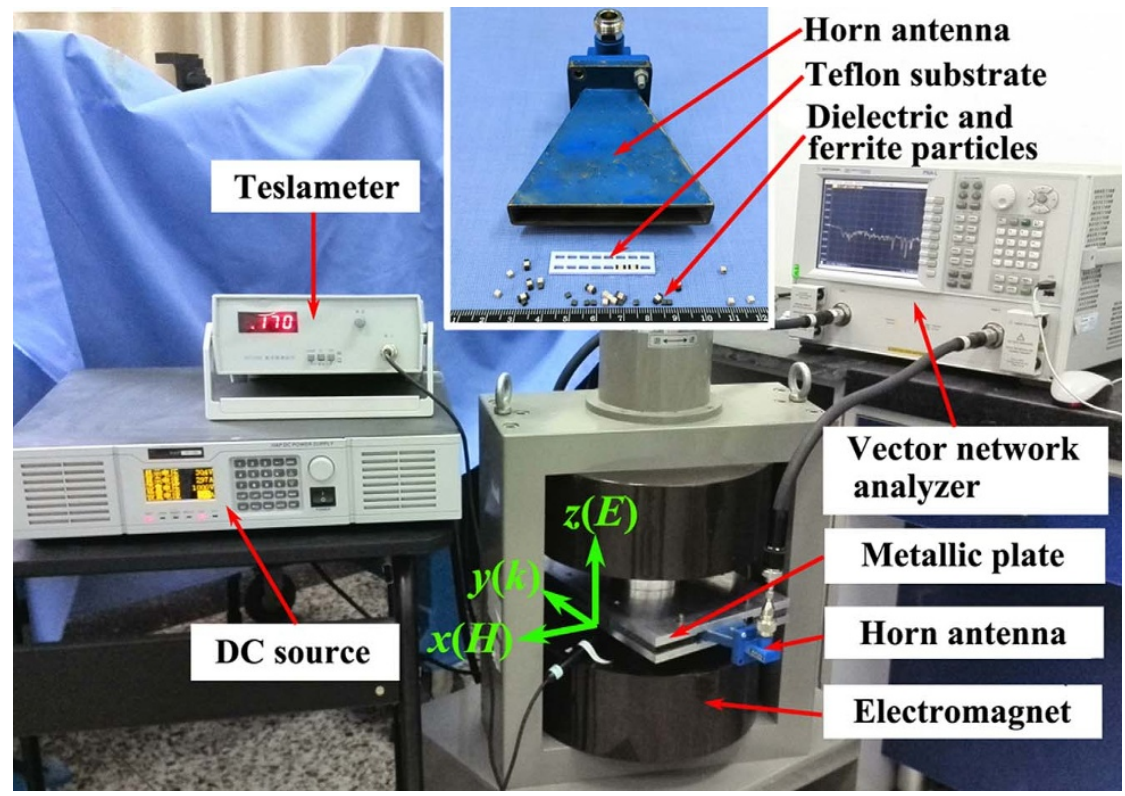

Figure 3 Experimental microwave setup. The sample is placed between two horn antennas. The propagation of the incident electromagnetic wave is along the $y$ axis, and the electric field and magnetic field are along the $z$ and $x$ axes, respectively. The bias magnetic field is generated by an electromagnet in the $z$ direction. The inset shows a photograph of the metamaterial.

and can be tuned by adjusting the input current. A teslameter is used to detect the magnetic field. The inset shows a photograph of the metamaterial and the horn antenna.

Experimental results. Figure 4a presents the experimental transmission spectra for the metamaterial under a series of applied magnetic fields $H$. First, a transmission dip induced by magnetic Mieresonance of dielectric cube occurs in the transmission spectrum for the metamaterial without applied magnetic field. Second, when a certain magnetic field is applied in the $z$ direction, two transmission dips appear in the range of $11-12 \mathrm{GHz}$. The resonance frequency of the transmission dip increases as $H$ increases, which exhibits a magnetically tunable behavior. Third, when $H$ is increased from 2500 Oe to 2600 Oe, the third transmission dip induced by ferromagnetic resonance of the ferrite cuboids appear in the range of $11-12 \mathrm{GHz}$. When $H=$ 0 , the ferrite cuboids are not magnetized and almost have no effect on the magnetic field distributions of the unit cells, as shown in the upper four maps in Fig. 1b. The typical magnetic Mieresonance is induced by the dielectric cube, which results in one transmission dip appeared in the spectra. The ferrite cuboids will be magnetized when $H>0$. By interacting with the electromagnetic wave, the ferromagnetic precession takes place in the ferrite cuboids. As shown in the lower four maps in Fig. 1b, the magnetic Mie-resonance in the dielectric cube is influenced by the ferromagnetic precession. Hence, two transmission dips appear in the transmission spectra. The ferromagnetic precession in the ferrite cuboids will enhance as $H$ increases. As a result, the resonance frequency of the transmission dip increases as $H$ increases.

The real parts of the effective permeabilities retrieved from the experimental scattering parameters under the same series of applied magnetic fields $H$ are depicted in Fig. $4 \mathrm{~b}$. The results reveal remarkable frequency dispersion in the range of 11-12 GHz in all cases. The number of the Lorentz-type dispersion in the range of $11-12 \mathrm{GHz}$ is one, two and three when $H=0$ Oe, 1500 Oe and 2500 Oe, respectively. The value of the effective permittivity is negative between the magnetic resonance frequency and the magnetic plasma frequency. Figure $4 \mathrm{c}$ shows the real parts of the effective permittivities retrieved from the experimental scattering parameters under the same series of applied magnetic fields $H$. The effective permittivity shows the antiresonance state. The electric resonance frequency increases as $H$ increases, thus, magnetically tunable behavior is demonstrated. From the analysis presented above, it is evident that the behavior of the experimental data is in good agreement with that of the simulated data.

\section{Discussion}

We experimentally and numerically demonstrated a magnetically tunable Mie resonance-based dielectric metamaterials. The tunable property is attributable to the coupling of the Mie resonance of dielectric cube and ferromagnetic precession of ferrite cuboid. When a certain magnetic field is applied, two or three resonance frequency dispersions appear in the range of 8-12 GHz, which indicates two or three peak values can be obtained. In particular, both the effective permittivity and permeability of the metamaterial can be tuned by adjusting the applied magnetic field. This work provides a new way to fabricate the tunable metamaterials, which has greater potential for wireless communication and radar technology.

\section{Methods}

Sample fabrication. The dielectric material chosen for this work was Barium Strontium Titanate (BST) ceramic. BST cubes were cut to dimensions of $2 \times 2 \times$ $2 \mathrm{~mm}^{3}$. The relative permittivity and dielectric loss are 120 and 0.015 , respectively. The ferrite material chosen for this work was yttrium iron garnet (YIG) ferrite. YIG cuboids were cut to dimensions of $2 \times 2 \times 1 \mathrm{~mm}^{3}$. The saturation magnetization $4 \pi M_{\mathrm{s}}$, linewidth $\Delta H$, and relative permittivity $\varepsilon_{\mathrm{r}}$ of the YIG rods were $1950 \mathrm{Gs}, 10 \mathrm{Oe}$, and 14.5 , respectively, and the same values were used in the simulations. The unit cell of the metamaterial is composed of one dielectric cube and two ferrite cuboids. Two ferrite cuboids were bonded with both ends of the dielectric cube. The sample was fabricated by inserting the dielectric cubes and ferrite cuboids into a Teflon substrate, as shown as an inset in Fig. 3. The distance between the dielectric cubes in the $x$ direction is the same as that in the $z$ direction $(5 \mathrm{~mm})$.

Simulations. The dimensions of the unit cell were $5 \times 5 \times 5 \mathrm{~mm}^{3}$. All the parameters of the dielectric cube and ferrite cuboid were the same as those in the experiments. A plane wave was assumed for the incident electromagnetic field, with polarization conditions corresponding to an electric field along the $x$ axis and a magnetic field along the $y$ axis. The bias magnetic field was applied in the $z$ direction. Numerical predictions of the transmission spectra were calculated using the commercial timedomain package CST Microwave Studio TM. 

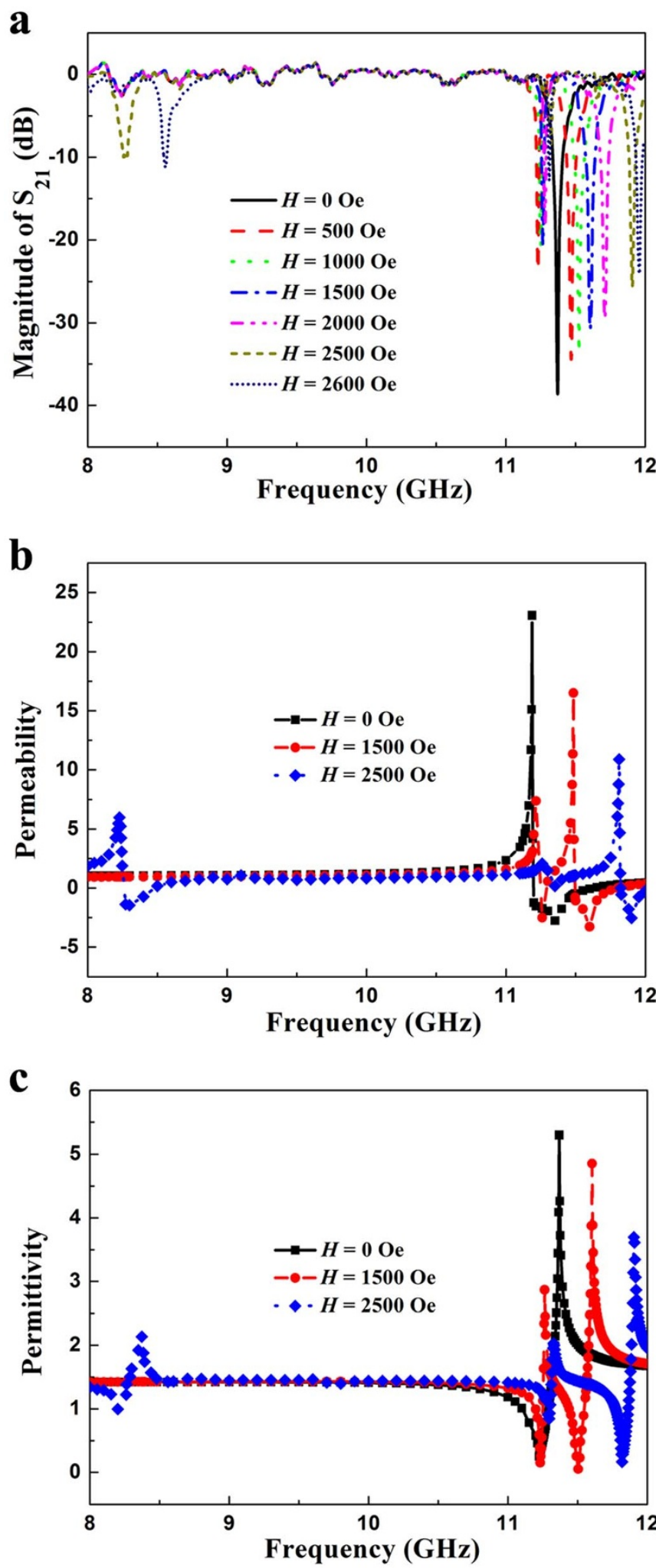

Figure $4 \mid$ Experimental results of the magnetically tunable Mie resonance-based dielectric metamaterial. (a) Experimental transmission spectra for the metamaterial under a series of applied magnetic fields $H$. Real parts of the effective (b) permeabilities and (c) permittivities retrieved from the experimental scattering parameters under a series of applied magnetic fields $H$.

Microwave measurements. The sample was placed between two horn waveguides connected to an HP 8720ES network analyzer, as shown in Fig. 3. The propagation of the incident electromagnetic wave was along the $y$ axis, and the electric field and magnetic field were along the $z$ and $x$ axes, respectively. The bias magnetic field provided by the electromagnets was applied in the $z$ direction.
1. Veselago, V. G. The electrodynamics of substance simultaneously negative values of $\varepsilon$ and $\mu$. Sov. Phys. Usp. 10, 509-514 (1968).

2. Pendry, J. B. Negative Refraction Makes a Perfect Lens. Phys. Rev. Lett. 85 3966-3969 (2000).

3. Schurig, D. et al. Metamaterial Electromagnetic Cloak at Microwave Frequencies. Science 314, 977-980, 1133628 (2006).

4. Yuan, G. et al. A broadband slab lens antenna formed from gradient refractive index metamaterials composed of cross-shaped cells. Microwave Opt. Technol. Lett. 56, 1124-1129 (2014).

5. Hoffman, A. J. et al. Negative refraction in semiconductor metamaterials. Nat. Mater. 6, 946-950 (2007).

6. Smith, D. R., Pendry, J. B. \& Wiltshire, M. C. K. Metamaterials and Negative Refractive Index. Science 305, 788-792 (2004).

7. Liu, M. et al. Terahertz-field-induced insulator-to-metal transition in vanadium dioxide metamaterial. Nature 487, 345-348 (2012).

8. Paul, T., Menzel, C., Rockstuhl, C. \& Lederer, F. Advanced Optical Metamaterials. Adv. Mater. 22, 2354-2357 (2010).

9. Gu, J. et al. Active control of electromagnetically induced transparency analogue in terahertz metamaterials. Nat. Commun. 3, 1151 (2012).

10. Ren, M. et al. Nanostructured Plasmonic Medium for Terahertz Bandwidth AllOptical Switching. Adv. Mater. 23, 5540-5544 (2011).

11. Chen, P.-Y., Farhat, M. \& Alù, A. Bistable and Self-Tunable Negative-Index Metamaterial at Optical Frequencies. Phys. Rev. Lett. 106, 105503 (2011).

12. Wu, Y., Lai, Y. \& Zhang, Z.-Q. Elastic Metamaterials with Simultaneously Negative Effective Shear Modulus and Mass Density. Phys. Rev. Lett. 107, 105506 (2011).

13. Liu, H., Zhao, X., Yang, Y., Li, Q. \& Lv, J. Fabrication of Infrared Left-Handed Metamaterials via Double Template-Assisted Electrochemical Deposition. Adv. Mater. 20, 2050-2054 (2008).

14. Kang, L. \& Lippens, D. Mie resonance based left-handed metamaterial in the visible frequency range. Phys. Rev. B 83, 195125 (2011).

15. Kuznetsov, A. I., Miroshnichenko, A. E., Fu, Y. H., Zhang, J. \& Luk'yanchuk, B. Magnetic light. Sci. Rep. 2, 492 (2012)

16. Zhao, Q. et al. Experimental Demonstration of Isotropic Negative Permeability in a Three-Dimensional Dielectric Composite. Phys. Rev. Lett. 101, 027402 (2008)

17. Vynck, K. et al. All-Dielectric Rod-Type Metamaterials at Optical Frequencies. Phys. Rev. Lett. 102, 133901 (2009).

18. Kim, J. \& Gopinath, A. Simulation of a metamaterial containing cubic high dielectric resonators. Phys. Rev. B 76, 115126 (2007).

19. Zhang, F., Kang, L., Zhao, Q., Zhou, J. \& Lippens, D. Magnetic and electric coupling effects of dielectric metamaterial. New J. Phys. 14, 033031 (2012).

20. Zhao, Q., Zhou, J., Zhang, F. L. \& Lippens, D. Mie resonance-based dielectric metamaterials. Mater. Today 12, 60-69 (2009).

21. Lewin, L. The electrical constants of a material loaded with spherical particles. Proc. Inst. Elec. Eng. 94, 65-68 (1947)

22. Zhao, H., Zhou, J., Kang, L. \& Zhao, Q. Tunable two-dimensional left-handed material consisting of ferrite rods and metallic wires. Opt. Express 17, 13373-13380 (2009).

23. Bi, K., Zhou, J., Zhao, H., Liu, X. \& Lan, C. Tunable dual-band negative refractive index in ferrite-based metamaterials. Opt. Express 21, 10746 (2013).

24. Smith, D. R., Schultz, S., Markoš, P. \& Soukoulis, C. M. Determination of effective permittivity and permeability of metamaterials from reflection and transmission coefficients. Phys. Rev. B 65, 195104 (2002).

25. Croënne, C., Fabre, B., Gaillot, D., Vanbésien, O. \& Lippens, D. Bloch impedance in negative index photonic crystals. Phys. Rev. B 77, 125333 (2008).

26. Chen, X., Grzegorczyk, T. M., Wu, B., Pacheco, J. \& Kong, J. A. Robust method to retrieve the constitutive effective parameters of metamaterials. Phys. Rev. E 70, 016608 (2004).

27. Koschny, T. et al. Impact of inherent periodic structure on effective medium description of left-handed and related metamaterials. Phys Rev B 71, 245105 (2005).

\section{Acknowledgments}

This work was supported by the National High Technology Research and Development Program of China under Grant No. 2012AA030403; the National Natural Science Foundation of China under Grant Nos. 51402163, 61376018, 61377067, 51032003, 11274198, 51102148 and 51221291; and the China Postdoctoral Research Foundation under Grant Nos. 2013M530042 and 2014T70075.

\section{Author contributions}

K.B. conceived the idea and designed experiments. K.B., Y.S.G. and X.M.L. performed the experiments. K.B., Q.Z., M.L. and J.H.X. developed the post-processing treatments of the experimental data. K.B. and Y.S.G. carried out numerical calculations and figures. K.B. and J.Z. wrote the paper. All authors contributed to scientific discussion and critical revision of the article. 


\section{Additional information}

Competing financial interests: The authors declare no competing financial interests.

How to cite this article: $\mathrm{Bi}, \mathrm{K}$. et al. Magnetically tunable Mie resonance-based dielectric metamaterials. Sci. Rep. 4, 7001; DOI:10.1038/srep07001 (2014).

(c) (1) () $\odot$ This work is licensed under a Creative Commons Attribution-NonCommercial-

gr wo No NoDerivs 4.0 International License. The images or other third party material in this article are included in the article's Creative Commons license, unless indicated otherwise in the credit line; if the material is not included under the Creative Commons license, users will need to obtain permission from the license holder in order to reproduce the material. To view a copy of this license, visit http:// creativecommons.org/licenses/by-nc-nd/4.0/ 